\title{
Synthesis of Novel C12-Nonmethylated Chlorophyll Derivatives from Methyl Pyropheophorbide- $a$ by Allomerization and Functionalization
}

\author{
Jin-Jun Wang, ${ }^{*}$ Peng Wang, Jia-Zhu Li, ${ }^{\dagger}$ Judit Jakus, ${ }^{\ddagger}$ and Young-Key Shim ${ }^{\dagger}$ \\ College of Chemistry and Chemical Engineering, Yantai University, Yantai 264005. *E-mail: wjj1955@163.com \\ ${ }^{\dagger} P D T$ Research Institute, School of Nano Engineering, Inje University, Gimhae 621-749, Korea \\ ¿Chemical Research Center, Hungarian Academy of Sciences, Budapest 1025, Pusztaszerj ut 59-67, Hungary \\ Received June 13, 2011, Accepted July 16, 2011
}

Key Words : Pyropheophorbide- $a$, Chlorophyll, Chlorin, Allomerization, Formylation, Functionalization

The major challenge in cancer therapy is the destruction of malignant cells while sparing the normal tissues. Among various choices for cancer treatment, photodynamic therapy (PDT) has a unique place and being noninvasive in nature makes it an increasingly desirable option. PDT is now widespread and is used clinically for treating various kinds cancers. ${ }^{1,2}$ Chlorins and bacteriochlorins, occurred in natural systems as a class of tetrapyrrolic macrocycles, have gained much interests in recent years because of their unique optical and photochemical properties which can be utilized as the second generation photosensitizers for PDT. ${ }^{3}$ Many naturally occurring (bacterio)chlorophylls $[=(\mathrm{B}) \mathrm{Chls}]$ have a highly reactive formyl group attached directly to their tetrapyrrole macrocycles. For example, each of Chl- $b$, Ch- $d$, $\mathrm{BCh}-f$ and $\mathrm{BCh}-e$ has a formyl group linked with its chlorin $\pi$-system at 3- or 7-position, respectively. ${ }^{4}$ It is well known that these carbon-oxygen double bonds, characterized their structural and spectroscopic properties, are readily modified to build various unique chemical structures by the typical chemical reactions of aromatic aldehyde, such as $\mathrm{C} 3-$ ethynylation with Bestmann-Ohira reagent in the presence of $\mathrm{Cs}_{2} \mathrm{CO}_{3},{ }^{5}$ synthesis of dimers via modified McMurry reaction using $\mathrm{TiCl}_{4}-\mathrm{Zn}$ combination, ${ }^{6}$ intramolecular cyclization in acid catalytic condition ${ }^{7}$ and $\mathrm{C} 3$-alkylation by Grignad reaction. ${ }^{8}$ Moreover, biosynthetic reduction of the formyl group at the 7-position in Chl- $b$ to the corresponding hydroxymethyl group has been observed in the course of its interconversion to Chl- $a$ possessing the 7-methyl group, ${ }^{9}$ which suggest that those formyl groups linked with chlorin chromophores play probably more important roles in vital movement. Therefore constructing formyl groups on the peripheries of chlorins and its functionalization have attracted a great deal of attention and became a very practical synthetic strategy for the preparation of chlorophyll- $a$ skeleton homologes with novel structures. Although many chlorins as potential photosensitizer for PDT have been synthesized by functionalization of formyl group attached directly to the periphery of chlorophyll derivatives, it was

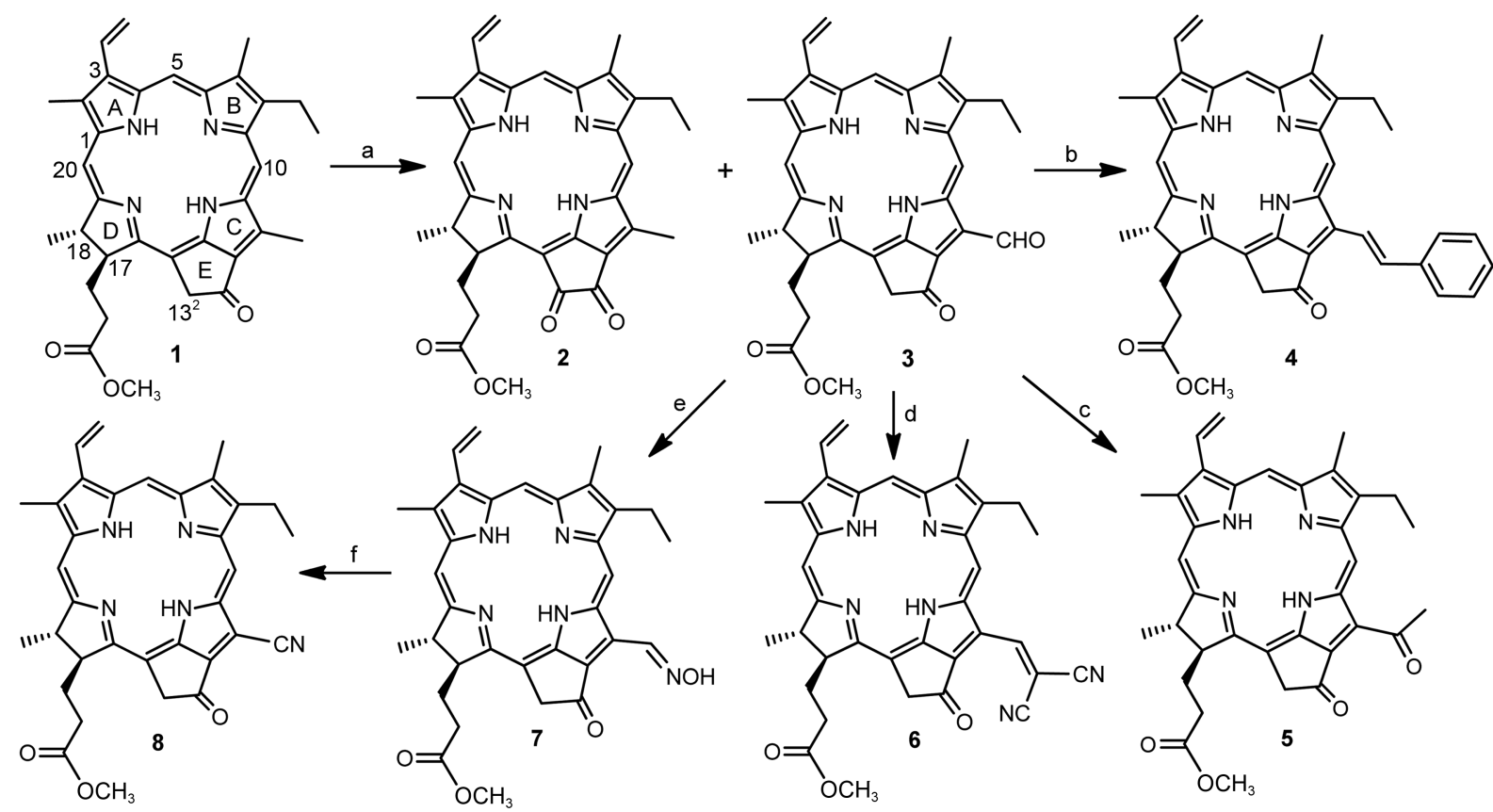

Scheme 1. Synthesis of C12-nonmethylated chlorophyll derivatives. Reagents and conditions: a) $\mathrm{LiOH} /$ air/ $\left./ \mathrm{THF} / \mathrm{H}_{2} \mathrm{O} ; \mathrm{b}\right) \mathrm{PhCH} 2 \mathrm{P}(\mathrm{Ph})_{3} \mathrm{Cl} /$ $\mathrm{NaOH} / \mathrm{CH}_{2} \mathrm{Cl}_{2} ;$ c) $\mathrm{CH}_{2} \mathrm{~N}_{2} / \mathrm{MeOH} / \mathrm{CH}_{2} \mathrm{Cl}_{2} ;$ d) $\mathrm{CNCH}_{2} \mathrm{CN} / \mathrm{N}(\mathrm{Et})_{3} ;$ e) $\mathrm{NH}_{2} \mathrm{OH} \cdot \mathrm{HCl} / \mathrm{MeOH} / \mathrm{CH}_{2} \mathrm{Cl} 2$; f) TCT/DMF. 
found that these chemical modifications mainly started at C3-position. Up to now, there are scarcely any reports on the functionalization at 12-position of pyropheophorbides. In order to systematically research and utilize such carbonyl groups to prepare new chlorins, we developed a synthetic route for the preparation of C12-nonmethyl-substituted chlorophyll derivatives from $\mathrm{C} 12$-formylchlorin, obtained firstly by allomerization of methyl pyropheophorbide- $a$ in this paper.

Methyl pyropheophorbide- $a$ 1 (MPP $a$ ) was used as starting material and successively treated with saturated methanol solution of $\mathrm{LiOH}$ in the presence of oxygen (exposure of the reaction mixture to air), acidified with $\mathrm{AcOH}$ and methylated with $\mathrm{CH}_{2} \mathrm{~N}_{2}$ to produce a mixture composed of numerous compounds. After careful step-wise separation, the $13^{2}$-keto methyl pyropheophorbide $a \mathbf{2}$ and 12-formyl methyl pyropheophorbide $a \mathbf{3}$ were obtained as major products in $28 \%$ and $17 \%$ yield, respectively. The formations of other chlorins in the allomerization, which could be as step-wise products, further demonstrate the reaction mechanisms of chlorophyll- $a$ homologues with triplet oxygen described previously. ${ }^{10}$ To prepare novel chlorins with different moieties joined at 12-position and improve their spectroscopic properties, the functionalization of the $\mathrm{C} 12$-formyl in $\mathbf{3}$ was examined because this $\mathrm{C}=\mathrm{O}$ is expected to allow versatile reactions to build novel structures on the periphery of chlorin. Unlike Wittig reaction of C3- and C8-formyl group, ${ }^{11}$ trans-styryl chlorin $\mathbf{4}$ as single Wittig product was obtained in $65 \%$ yield by reaction with benzyltriphenylphosphonium chloride in dichloromethane in the presence of $\mathrm{NaOH}$. Lack of cis-isomer forming in this procedure may be due to stronger steric repulsion between the cis-phenyl group and carbonyl group at $13^{1}$-position. The formyl of $\mathbf{3}$, upon being subjected to the Büchner-CurtiusSchlotterbeck reaction by treating with ethereal diazomethane at $0{ }^{\circ} \mathrm{C}$ for $24 \mathrm{~h}$, was converted into acetyl group as an exclusive product to afford chlorin $\mathbf{5}$ in $78 \%$ yield. The

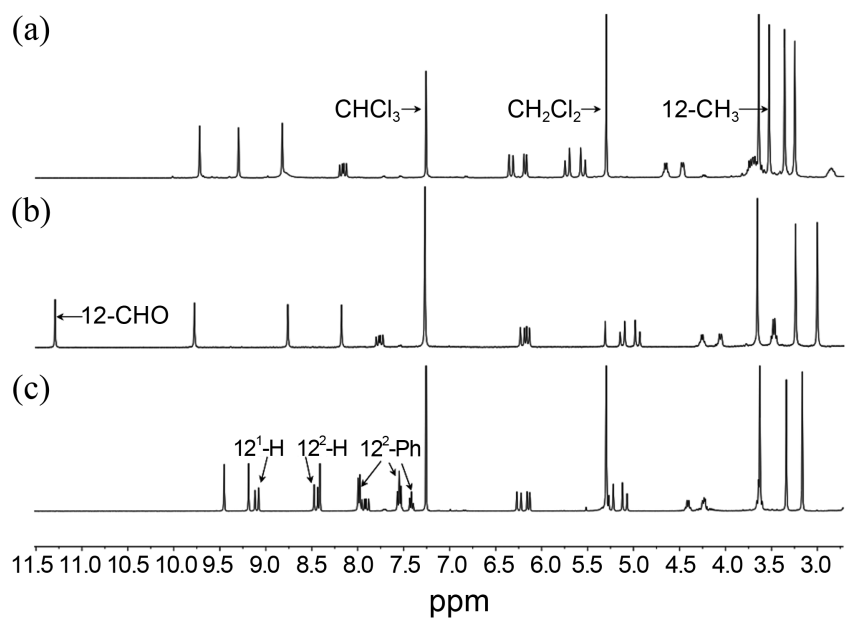

Figure 1. The comparative ${ }^{1} \mathrm{H}$ NMR spectra $\left(\mathrm{CDCl}_{3}, 400 \mathrm{MHz}\right)$ in the region $\delta 2.7-11.5 \mathrm{ppm}$ of (a) MPPa 1, (a) 12-devinyl-12-formyl pyropheophorbide $a$ methyl ester $\mathbf{3}$, and (c) styryl substituted derivative 4.
Knoevenagel condensations of the $\mathbf{3}$ with malononitrile were performed in $\mathrm{CH}_{2} \mathrm{Cl}_{2}$ in the presence of triethylamine at room temperature to form 12-disubstituted methylenechlorin 6 in moderate yield. Aldehydeoxime 7, easily prepared from 3 by condensation with hydroxylamine hydrochloride, underwent the Beckmann rearrangement upon treatment with 2,4,6-trichloro-[1,3,5]triazine (TCT) in DMF at room temperature to afford C12-cyanidated chlorin $\mathbf{8}$ in excellent yields (Scheme 1). The structure of the new chlorins was determined by ${ }^{1} \mathrm{H}$ NMR, MS, Uv-vis spectra and elemental analysis. ${ }^{12-17}$

In the ${ }^{1} \mathrm{H}$ NMR spectra of all the C12-nonmethyl substituted chlorins, their various typical signals of the moiety attached on 12-position, such as formyl, acetyl, vinyl, hydroxyimino group and so on, can be ascribed reasonably (Fig. 1). It was found that only three sets of singlet signals of methyl groups bonded to their peripheries were discovered between 2.71-3.68 ppm (Fig. 1(b) and (c)), and other chemical shifts indicated that the original structures were still intact. The ${ }^{1} \mathrm{H}$ NMR spectra of compound 4 showed clearly the two vinylic proton signals with large-coupling constant ( $\delta=9.10$ and $8.14, J=15.4 \mathrm{~Hz}$ ) as typical of an $E$ geometry of the double bond (Fig. 1(c)), suggesting that the Wittig reaction of C-12 formyl group only formed the $E$ isomer.

In the Uv-vis spectra, the position and intensity of Soret band as well as $\mathrm{Q}_{\mathrm{y}}$ bands of the final products were changed intensively. The introduction of formyl group at 12-position of MPPa 1 causes an obvious bathochromic shift of the $\mathrm{Q}_{\mathrm{y}}$ peak maxima from 668 to $690 \mathrm{~nm}$ in dichloromethane (Fig. 2). In contrast to $\mathrm{C} 3$-formylation, which shifted the peak positions of Qy bands to $694 \mathrm{~nm},{ }^{18}$ the bathochromic effect resulted from introduction of $12-\mathrm{C}=\mathrm{O}$ is relatively low due to the repellency with $\mathrm{C} 13^{1}$-carbonyl group to disrupt the coplaner with chlorin chromophore and weaken their conjugate interaction. Another cause forming a shorter redshifting may be that the 12-formyl group can not efficiently extend macrocycle $\pi$-system like C3-formyl group which form the most longest conjugated region with carbonoxygen double bond at $13^{1}$-position. It was found that all the

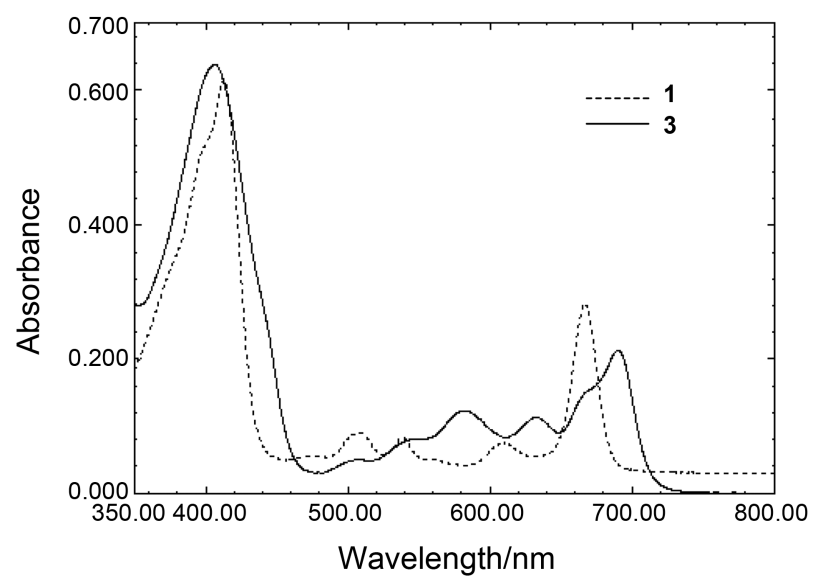

Figure 2. Visible spectra of $\operatorname{MPP} a \mathbf{1}$ and methyl 12-formyl-12demethyl-pheophorbide- $a \mathbf{3}$ in $\mathrm{CH}_{2} \mathrm{Cl}_{2}$. 
Table 1. Absorption properties of C12-nonmethylated chlorophyll derivatives. $\Delta$ Soret and $\Delta \mathrm{Q}_{\mathrm{y}}$ represent the change of the Soret band and $\mathrm{Q}_{\mathrm{y}}$ band between the C12-nonmethylated chlorin and their start material $\mathrm{MPP} a$

\begin{tabular}{ccccc}
\hline \multirow{2}{*}{ Compound } & \multicolumn{4}{c}{ Absorption $\lambda_{\max }(\mathrm{nm})$ (relative intensity) } \\
\cline { 2 - 5 } & Soret & $\Delta$ Soret & $\mathrm{Q}_{\mathrm{y}}$ & $\Delta \mathrm{Q}_{\mathrm{y}}$ \\
\hline $\mathbf{1}$ & $414(1.00)$ & 0 & $668(0.38)$ & 0 \\
$\mathbf{3}$ & $410(1.00)$ & -4 & $690(0.45)$ & 22 \\
$\mathbf{4}$ & $410(1.00)$ & -4 & $680(0.37)$ & 12 \\
$\mathbf{5}$ & $407(1.00)$ & -7 & $685(0.40)$ & 17 \\
$\mathbf{6}$ & $401(1.00)$ & -13 & $699(0.12)$ & 31 \\
$\mathbf{7}$ & $406(1.00)$ & -7 & $689(0.49)$ & 21 \\
$\mathbf{8}$ & $401(1.00)$ & -13 & $670(0.28)$ & 2 \\
\hline
\end{tabular}

Table 2. $\mathrm{IC}_{50}$ results of the nonmethylated chlorins on mouse sarcoma S-180 cell line

\begin{tabular}{cccccccc}
\hline Compound & $\mathbf{1}$ & $\mathbf{3}$ & $\mathbf{4}$ & $\mathbf{5}$ & $\mathbf{6}$ & $\mathbf{7}$ & $\mathbf{8}$ \\
\hline $\mathrm{IC}_{50}(\mu \mathrm{M})$ & 6.812 & 0.430 & 2.125 & 2.875 & 0.601 & 0.102 & 3.420 \\
\hline
\end{tabular}

C12-nonmethylated derivatives 3-8 demonstrated regularly hypsochromic shifts of their Soret bands (4-13 nm) and bathochromic shifts of their $\mathrm{Q}_{\mathrm{y}}$ bands $(2-31 \mathrm{~nm})$ in various degrees compared to their start materials 2 , respectively. For example, the dicyanomethylene substituted derivative $\mathbf{6}$ showed a Soret band at $401 \mathrm{~nm}$ with $13 \mathrm{~nm}$ hypsochromic shift and a $\mathrm{Q}_{\mathrm{y}}$ band at $699 \mathrm{~nm}$ with $31 \mathrm{~nm}$ bathochromic shift, respectively. Similar relationships were also discovered for the other C12-nonmethylated derivatives (Table 1).

Preliminary in vitro photodynamic effects of these derivatives were examined on mouse sarcoma S-180 cell line. Table 2 shows the $\mathrm{IC}_{50}$ values of these new photosensitizers on mouse sarcoma S-180 cell line after PDT. The reference compound MPPa 1 showed a relative low effect after PDT $\left(\mathrm{IC}_{50}=6.812 \mu \mathrm{M}\right)$, while all the tested C12-nonmethylated derivatives showed relatively high PDT effect than the reference compound MPPa 1. Among them, the hydroxyimino-substituted chlorin 7 showed the highest PDT effect $\left(\mathrm{IC}_{50}=0.102 \mu \mathrm{M}\right)$. Furthermore, we are aiming to explore, in greater depth, the other biological effects of these compounds for PDT.

In conclusion, we have successfully synthesized methyl 12-formylpyropheophorbide- $a$ by allomerization from MPPa. A series of C12-nonmethyl-substituted chlorophyll derivatives were conveniently prepared from this C12formyl chlorin by common reactions. Their PDT effects on mouse sarcoma S-180 cell line were also examined, it was found that all the C12-nonmethylated chlorins showed higher PDT anticancer effect than those of MPP $a$. Formyl group possessing highly reactivity provides an excellent reactive site and broadens synthetic approaches for designing novel chlorins with extended $\pi$-conjugation system at 12-position. The research on making use of the C12-functionalized chlorins for constructing other special structure on their peripheries is currently in progress.
Acknowledgments. The authors appreciate the financial support by the natural science foundation of Shandong Province of China (No. Y2008B49) and Science and Technology Cooperation Project of China and Hungary (No. 2008-333)

\section{References and Notes}

1. (a) Dolmans, D. E.; Fukumura, D.; Jain, R. K. Nat. Rev. Cancer 2003, 3, 380-387. (b) Brown, S. B.; Brown, E. A.; Walker, I. Lancet Oncol. 2004, 5, 497-508.

2. (a) Pandey, R. K.; Zheng, G. In Porphyrin Handbook; Smith, K. M., Kadish, K., Guilard, R., Eds.; Academic Press: San Diego, 2000; Vol. 6. (b) MacDonald, I. J.; Dougherty, T. J. J. Porphyrins Phthalocyanines 2001, 5, 105-129.

3. (a) Nyman, E. S.; Hynninen, P. H. J. Photochem. Photobiol. B. 2004, 73, 1-28. (b) Bourre, L.; Simonneaux, G.; Farand, Y.; Thibautt, S.; Lajat, Y.; Patrice, T. J. Photochem. Photobiol. B 2003, 69, 179-192.

4. (a) Scheer, H. In Light HarVesting Antennas in Photosynthesis; Green, B. R., Parson, W. W., Eds.; Kluwer Academic Publisher: Dordrecht, The Netherlands, 2003; pp 29-81. (b) Miyashita, H.; Adachi, K.; Kurano, N.; Ikemoto, H.; Chihara, M.; Miyachi, S. Plant Cell Physiol. 1997, 38, 274-281. (c) Chen, Y. H.; Li, G. L.; Pandey, R. K. Curr. Org. Chem. 2004, 8, 1105-1134.

5. Sasaki, S.; Mizutani, K.; Kunieda, M.; Tamiaki, H. Tetrahedron Lett. 2008, 49, 4113-4115.

6. Goswami, L. N.; Ethirajan, M.; Mahabeer, P.; Dobhal, M. P.; Min Zhang, M.; Missert, J. R.; Masayuki Shibata, M.; Karl, M.; Kadish, K. M.; Ravindra, K.; Pandey, R. K. J. Org. Chem. 2009, 74, 568-579.

7. Wang, J. J.; Wu, X. R.; Shim, Y. K. Chin. J. Chem. 2005, 23, 7680

8. Wang, J. J.; Shim, Y. K.; Jiang, G. J.; Imafuku, K. J. Heterocyclic. Chem. 2004, 41, 29-35.

9. Tanaka, A.; Tanaka, R. Curr. Opin. Plant Biol. 2006, 9, 248.

10. (a) Zhang, G.; Potter, W. R.; Camacho, S. H.; Misser, J. R.; Wang, G. S.; Bellinier, D. A.; Henderson, B. W.; Rodgers, M. A. J.; Dougherty, T. J.; Pandey, R. K. J. Med. Chem. 2001, 44, 15401559. (b) Hynninen, P. H.; Leppäkases, T. S.; Mesilaakso, M. Tetrahedron Lett 2006, 47, 1663-1668.

11. (a) Tamiaki, H.; Kouraba, M. Tetrahedron 1997, 53, $10677-$ 10688. (b) Wang, J. J.; Imafuku, K.; Shim, Y. K.; Jiang, G. J. J. Heterocyclic. Chem. 2005, 42, 835-840. (c) Sasaki, S. S.; Omoda, M.; Tamiaki, H. J. Photochem Photobiol (A) 2004, 162, 307-315.

12. The procedure for the allomerization of MPPa: To a THF solution $(25 \mathrm{~mL})$ of 1 (514 $\mathrm{mg}, 0.937 \mathrm{mmol})$, an aqueous solution $(5 \mathrm{~mL})$ of $\mathrm{LiOH}(1.2 \mathrm{~g})$ and methanol $(15 \mathrm{~mL})$ was sequentially added. This mixture was violently stirred in open system in dark for $3 \mathrm{~h}$, poured into cool water, adjusted $\mathrm{pH}$ to 3 with sulfuric acid and then extracted with dichloromethane $(2 \times 50 \mathrm{~mL})$. The combined extracts was washed with water, dried over anhydrous $\mathrm{Na}_{2} \mathrm{SO}_{4}$ and treated with $\mathrm{CH}_{2} \mathrm{~N}_{2}$ for short time (approximately $5 \mathrm{~min}$ ). After evaporation in vacuo, the residue was purified on chromatographe on a silica gel column with hexane-ethyl acetate (5:1) to give 2 (147 $\mathrm{mg}, 28 \%$ ) as a yellow solid, 3 (90 mg, 17\%) as a bluish-green solid. 3: UV-vis $\left(\mathrm{CHCl}_{3}\right) \lambda_{\text {max }}$ : 410 (relative intensity, 1.00), $556(0.15), 580(0.25), 633(0.22), 690(0.35) \mathrm{nm}$; ${ }^{1} \mathrm{H}$ NMR $\left(400 \mathrm{MHz}, \mathrm{CDCl}_{3}\right) \delta 0.07,0.14$ (each br s $\left.2 \mathrm{H}, \mathrm{NH}\right), 1.59$ $\left(\mathrm{t}, J=7.6 \mathrm{~Hz}, 8-\mathrm{CH}_{3}\right), 1.75\left(\mathrm{~d}, J=7.3 \mathrm{~Hz}, 18-\mathrm{CH}_{3}\right), 2.18-2.26$, 2.32-2.40, 2.52-2.63 (each $\mathrm{m}$, all 4H, 17a + 17b-H), 2.99, 3.23, 3.64 (each $3 \mathrm{H}$, each s, $\left.\mathrm{CH}_{3}+\mathrm{OCH}_{3}\right), 3.46$ (q, $\left.J=7.6 \mathrm{~Hz}, 8 \mathrm{a}-\mathrm{H}\right)$, 4.04 (d, $J=8.8 \mathrm{~Hz}, 1 \mathrm{H}, 18-\mathrm{H}), 4.24$ (q, $J=7.1 \mathrm{~Hz}, 1 \mathrm{H}, 17-\mathrm{H})$, $4.95\left(\mathrm{~d}, J=20.0 \mathrm{~Hz}, 1 \mathrm{H}, 13^{2}-\mathrm{H}\right), 5.11\left(\mathrm{~d}, J=20.0 \mathrm{~Hz}, 1 \mathrm{H}, 13^{2}-\mathrm{H}\right)$, 6.12 (d, $J=11.6 \mathrm{~Hz}, 1 \mathrm{H}$, cis-3b-H), 6.20 (d, $J=18.0 \mathrm{~Hz}, 1 \mathrm{H}$, trans-3b-H), 7.75 (dd, $J=17.9,11.6 \mathrm{~Hz}, 3 \mathrm{a}-\mathrm{H}), 8.17,8.75,9.77$ 
(each 1H, s, meso-H), 11.28 (s, 1H, 12-CHO); EI-MS m/z: 563.4 $\left(\mathrm{MH}^{+}\right)$; Anal. calcd for $\mathrm{C}_{34} \mathrm{H}_{34} \mathrm{~N}_{4} \mathrm{O}_{4}$ : C 72.58, H 6.09, N 9.96; found $\mathrm{C} 72.68, \mathrm{H} 6.17, \mathrm{~N} 9.73$

13. Methyl 12-styryl-12-demethylpyropheobide-a 4: Chlorin 3 $(100 \mathrm{mg}, 0.178 \mathrm{mmol})$ and benzyltriphenylphosphonium chloride (100 mg, $0.257 \mathrm{mmol}$ ) were dissolved in $50 \mathrm{~mL} \mathrm{CH}_{2} \mathrm{Cl}_{2}$ and a solution of $\mathrm{NaOH}(40 \mathrm{mg})$ in $\mathrm{H}_{2} \mathrm{O}(15 \mathrm{~mL})$ was added with stirring. The solution was stirred at room temperature under $\mathrm{N}_{2}$ for $1 \mathrm{~h}$ and poured into ice water and $\mathrm{CH}_{2} \mathrm{Cl}_{2}$. The aqueous phase was extracted with several portions of $\mathrm{CH}_{2} \mathrm{Cl}_{2}$ and the combined organic phases were washed with aq. $2 \% \mathrm{HCl}$, aq. $4 \% \mathrm{NaHCO}_{3}$ and water, dried over $\mathrm{Na}_{2} \mathrm{SO}_{4}$ and evaporated in vacuo to dryness. The residue was purified on chromatographe on a silica gel column with hexane-ethyl acetate (3:1) to give $4(62 \mathrm{mg}, 65 \%)$ as a bluish-green solid. UV-vis $\left(\mathrm{CHCl}_{3}\right) \lambda_{\max }: 410$ (relative intensity, 1.00), 510 (0.09), 542 (0.09), $610(0.05), 680(0.37) \mathrm{nm} ;{ }^{1} \mathrm{H}$ NMR $\left(\mathrm{CDCl}_{3}\right) \delta-1.09,0.82($ each br s, $2 \mathrm{H}, \mathrm{NH}), 1.67(\mathrm{t}, J=7.6 \mathrm{~Hz}, 3 \mathrm{H}$, $\left.8 \mathrm{~b}-\mathrm{CH}_{3}\right), 1.81$ (d, $\left.J=7.3 \mathrm{~Hz}, 3 \mathrm{H}, 18-\mathrm{CH}_{3}\right), 2.25-2.36,2.51-2.61$, 2.62-2.73 (each m, all 4H, 17a-H + 17b-H), 3.17, 3.34, 3.63 (each s, each $\left.3 \mathrm{H}, \mathrm{CH}_{3}+\mathrm{OCH}_{3}\right), 3.64(\mathrm{q}, J=7.6 \mathrm{~Hz}, 2 \mathrm{H}, 8 \mathrm{a}-\mathrm{H}), 4.23(\mathrm{dd}$, $J=11.8,6.3 \mathrm{~Hz}, 1 \mathrm{H}, 17-\mathrm{H}), 4.41$ (q, $J=7.3 \mathrm{~Hz}, 1 \mathrm{H}, 18-\mathrm{H}), 5.09$ $\left(\mathrm{d}, J=19.7 \mathrm{~Hz}, 1 \mathrm{H}, 13^{2}-\mathrm{H}\right), 5.24\left(\mathrm{~d}, J=19.7 \mathrm{~Hz}, 1 \mathrm{H}, 13^{2}-\mathrm{H}\right)$, 6.14 (dd, $J=11.5,1.0 \mathrm{~Hz}, 1 \mathrm{H}$, cis-3b-H), 6.25 (dd, $J=17.9,1.0$ $\mathrm{Hz}, 1 \mathrm{H}$, trans-3b-H), $7.42(\mathrm{t}, J=7.3 \mathrm{~Hz}, 1 \mathrm{H}, \mathrm{Ph}-\mathrm{H}), 7.55(\mathrm{t}, J=$ $7.3 \mathrm{~Hz}, 2 \mathrm{H}, \mathrm{Ph}-\mathrm{H}), 7.91(\mathrm{dd}, J=17.9,11.5 \mathrm{~Hz}, 1 \mathrm{H}, 3 \mathrm{a}-\mathrm{H}), 7.98(\mathrm{t}$, $J=7.5 \mathrm{~Hz}, 2 \mathrm{H}, \mathrm{Ph}-\mathrm{H}), 8.46(\mathrm{~d} J=15.8 \mathrm{~Hz}, 1 \mathrm{H}, 12 \mathrm{~b}-\mathrm{H}), 9.10(\mathrm{~d}, J$ $=15.8 \mathrm{~Hz}, 1 \mathrm{H}, 12 \mathrm{a}-\mathrm{H}), 8.41,9.19,9.45($ each $\mathrm{s}$, each $1 \mathrm{H}$, mesoH); EI-MS m/z: $637.4\left(\mathrm{MH}^{+}\right)$; Anal. calcd for $\mathrm{C}_{41} \mathrm{H}_{40} \mathrm{~N}_{4} \mathrm{O}_{3}$ : C 77.33, H 6.33, N 8.80; found C 77.18, H 6.11, N 8.69.

14. Methyl 12-acetyl-12-demethylpyropheobide-a 5: Chlorin 3 (46 $\mathrm{mg}, 0.082 \mathrm{mmol}$ ) was dissolved in ethereal diazomethane $(20 \mathrm{~mL})$ and placed in refrigerator for $12 \mathrm{~h}$. The resulting mixture was evaporated to dryness. The residue was purified on chromatographe on a silica gel column with hexane-ethyl acetate (4:1) to give $5(27 \mathrm{mg}, 58 \%)$ as a green solid. UV-vis $\left(\mathrm{CHCl}_{3}\right) \lambda_{\max }: 407$ (relative intensity, 1.00), $506(0.05), 575(0.19), 628(0.17), 685$ $(0.40) \mathrm{nm} ;{ }^{1} \mathrm{H}$ NMR $\left(\mathrm{CDCl}_{3}\right) \delta 0.12,0.83$ (each br s, $\left.2 \mathrm{H}, \mathrm{NH}\right)$, $1.60\left(\mathrm{t}, J=7.6 \mathrm{~Hz}, 3 \mathrm{H}, 8 \mathrm{~b}-\mathrm{CH}_{3}\right), 1.74\left(\mathrm{~d}, J=7.7 \mathrm{~Hz}, 3 \mathrm{H}, 18-\mathrm{CH}_{3}\right)$, 2.21-2.24, 2.30-2.36, 2.51-2.60 (each m, all $4 \mathrm{H}, 17 \mathrm{a}-\mathrm{H}+17 \mathrm{~b}-\mathrm{H}$ ), $3.01,3.23,3.41,3.63$ (each s, each $\left.3 \mathrm{H}, \mathrm{CH}_{3}+\mathrm{OCH}_{3}\right), 3.50$ (q, $J=$ $7.6 \mathrm{~Hz}, 2 \mathrm{H}, 8 \mathrm{a}-\mathrm{H}), 4.06(\mathrm{~d}, J=8.8 \mathrm{~Hz}, 1 \mathrm{H}, 17-\mathrm{H}), 4.24(\mathrm{q}, J=7.4$ $\mathrm{Hz}, 1 \mathrm{H}, 18-\mathrm{H}), 4.98\left(\mathrm{~d}, J=20.0 \mathrm{~Hz}, 1 \mathrm{H}, 13^{2}-\mathrm{H}\right), 5.15(\mathrm{~d}, J=20.0$ $\left.\mathrm{Hz}, 1 \mathrm{H}, 13^{2}-\mathrm{H}\right), 6.13(\mathrm{~d}, J=11.5 \mathrm{~Hz}, 1 \mathrm{H}$, cis-3b-H), 6.19 (d, $J=$ $17.8 \mathrm{~Hz}, 1 \mathrm{H}$, trans-3b-H), $7.76(\mathrm{dd}, J=17.8,11.5 \mathrm{~Hz}, 1 \mathrm{H}, 3 \mathrm{a}-\mathrm{H})$, 8.17, 8.79, 10.06 (each s, each $1 \mathrm{H}$, meso-H); EI-MS $m / z: 577.4$ $\left(\mathrm{MH}^{+}\right)$; Anal. calcd for $\mathrm{C}_{35} \mathrm{H}_{36} \mathrm{~N}_{4} \mathrm{O}_{4}$ : C 72.90, H 6.29, N 9.72; found $\mathrm{C} 72.87, \mathrm{H} 6.43, \mathrm{~N} 9.59$

15. Methyl 12-( $\beta, \beta$-dicyanomethylene)-12-demethylpyropheobidea 6: Chlorin 3 (66 $\mathrm{mg}, 0.117 \mathrm{mmol}$ ) was dissolved in THF (30 $\mathrm{mL}$ ) and malononitrile $(120 \mathrm{mg})$ and triethylamine $(0.3 \mathrm{~mL})$ were added with stirring. The resulting mixture was stirred at room temperature in dark for $8 \mathrm{~h}$ and poured into ice water and $\mathrm{CH}_{2} \mathrm{Cl}_{2}$. The aqueous phase was extracted with several portions of $\mathrm{CH}_{2} \mathrm{Cl}_{2}$ and the combined organic phases were washed with aq. $4 \%$ $\mathrm{NaHCO}_{3}$ and water, dried over $\mathrm{Na}_{2} \mathrm{SO}_{4}$ and evaporated in vacuo to dryness. The residue was purified on chromatographe on a silica gel column with hexane-ethyl acetate $(5: 1)$ to give $6(65 \%)$ as a green solid. UV-vis $\left(\mathrm{CHCl}_{3}\right) \lambda_{\max }$ : 401 (relative intensity, $1.00), 504(0.08), 624(0.09), 658(0.25), 699(0.12) \mathrm{nm} ;{ }^{1} \mathrm{H}$ NMR $\left(\mathrm{CDCl}_{3}\right) \delta 0.09,0.68($ each br s, $2 \mathrm{H}, \mathrm{NH}), 1.48(\mathrm{t}, J=7.5 \mathrm{~Hz}, 3 \mathrm{H}$, $\left.8 \mathrm{~b}-\mathrm{CH}_{3}\right), 1.78\left(\mathrm{~d}, J=7.3 \mathrm{~Hz}, 3 \mathrm{H}, 18-\mathrm{CH}_{3}\right), 2.11-2.21,2.28-2.40$,
2.51-2.62 (each m, all 4H, 17a-H + 17b-H), 2.94, 3.23, 3.66 (each s, each $\left.3 \mathrm{H}, \mathrm{CH}_{3}+\mathrm{OCH}_{3}\right), 4.03(\mathrm{q}, J=7.5 \mathrm{~Hz}, 2 \mathrm{H}, 8 \mathrm{a}-\mathrm{H}), 4.05(\mathrm{~d}$, $J=8.4 \mathrm{~Hz}, 1 \mathrm{H}, 17-\mathrm{H}), 4.23(\mathrm{q}, J=7.5,1.9 \mathrm{~Hz}, 1 \mathrm{H}, 18-\mathrm{H}), 4.90(\mathrm{~d}$, $\left.J=19.9 \mathrm{~Hz}, 1 \mathrm{H}, 13^{2}-\mathrm{H}\right), 5.05\left(\mathrm{~d}, J=19.9 \mathrm{~Hz}, 1 \mathrm{H}, 13^{2}-\mathrm{H}\right), 6.15$ $\left(\mathrm{d}, J=11.6 \mathrm{~Hz}, 1 \mathrm{H}, c_{i s}-3 \mathrm{~b}-\mathrm{H}\right), 6.20(\mathrm{~d}, J=17.9 \mathrm{~Hz}, 1 \mathrm{H}$, trans-3b$\mathrm{H}), 7.72(\mathrm{dd}, J=17.9,11.6 \mathrm{~Hz}, 1 \mathrm{H}, 3 \mathrm{a}-\mathrm{H}), 8.53(\mathrm{~s}, 1 \mathrm{H}, 12 \mathrm{a}-\mathrm{H})$, $8.21,8.64,8.69$ (each $\mathrm{s}$, each $1 \mathrm{H}$, meso-H); EI-MS $m / z: 611.4$ $\left(\mathrm{MH}^{+}\right)$, Anal. calcd for $\mathrm{C}_{37} \mathrm{H}_{34} \mathrm{~N}_{6} \mathrm{O}_{3}$ : C 72.77, H 5.61, N 13.76; found $\mathrm{C} 72.90, \mathrm{H} 5.76, \mathrm{~N} 13.77$.

16. Methyl 12-hydroxyimino-12-demethylpyropheobide-a 7: Chlorin $3(88 \mathrm{mg}, 0.156 \mathrm{mmol}$ ) was dissolved in $10 \mathrm{~mL} \mathrm{MeOH}$ and a solution of hydroxylamine hydrochloride $(140 \mathrm{mg})$ in $\mathrm{MeOH}(10$ $\mathrm{mL})$ and concentrated $\mathrm{HCl}(0.1 \mathrm{~mL})$ were added with stirring. The resulting mixture was stirred at room temperature for $2 \mathrm{~h}$ and poured into ice water and $\mathrm{CH}_{2} \mathrm{Cl}_{2}$. The aqueous phase was extracted with several portions of $\mathrm{CH}_{2} \mathrm{Cl}_{2}$ and the combined organic phases were washed with aq. $4 \% \mathrm{NaHCO}_{3}$ and water, dried over $\mathrm{Na}_{2} \mathrm{SO}_{4}$ and evaporated in vacuo to dryness. The residue was purified on chromatographe on a silica gel column with hexane-ethyl acetate $(3: 1)$ to give $7(74 \mathrm{mg}, 82 \%)$ as a green solid. UV-vis $\left(\mathrm{CHCl}_{3}\right) \lambda_{\max }: 406$ (relative intensity, 1.00), 523 (0.06), $565(0.20), 629(0.16), 689(0.49) \mathrm{nm} ;{ }^{1} \mathrm{H} \mathrm{NMR}\left(\mathrm{CDCl}_{3}\right) \delta$ $-1.32,-1.10$ (each br s, 2H, NH), 1.37 (t, $J=7.6 \mathrm{~Hz}, 3 \mathrm{H}, 8 \mathrm{~b}-$ $\left.\mathrm{CH}_{3}\right), 1.82\left(\mathrm{~d}, J=7.3 \mathrm{~Hz}, 3 \mathrm{H}, 18-\mathrm{CH}_{3}\right), 2.31-2.36,2.57-2.76$ (each m, all 4H, 17a-H + 17b-H), 2.71, 3.27, 3.68 (each s, each $\left.3 \mathrm{H}, \mathrm{CH}_{3}+\mathrm{OCH}_{3}\right), 2.96(\mathrm{q}, J=7.6 \mathrm{~Hz}, 2 \mathrm{H}, 8 \mathrm{a}-\mathrm{H}), 4.17(\mathrm{~d}, J=8.6$ $\mathrm{Hz}, 1 \mathrm{H}, 17-\mathrm{H}), 4.39$ (q, $J=7.3 \mathrm{~Hz}, 1 \mathrm{H}, 18-\mathrm{H}), 5.01$ (d, $J=19.6$ $\left.\mathrm{Hz}, 1 \mathrm{H}, 13^{2}-\mathrm{H}\right), 5.22\left(\mathrm{~d}, J=19.6 \mathrm{~Hz}, 1 \mathrm{H}, 13^{2}-\mathrm{H}\right), 6.08(\mathrm{~d}, J=11.4$ $\mathrm{Hz}, 1 \mathrm{H}$, cis-3b-H), 6.15 (d, $J=18.2 \mathrm{~Hz}, 1 \mathrm{H}$, trans-3b-H), 7.71 $(\mathrm{dd}, J=18.2,11.4 \mathrm{~Hz}, 1 \mathrm{H}, 3 \mathrm{a}-\mathrm{H}), 8.95(\mathrm{~s}, 1 \mathrm{H}, 12 \mathrm{a}-\mathrm{H}), 8.30,8.57$, 9.51 (each s, each $1 \mathrm{H}$, meso-H); EI-MS $m / z$ : $578.3\left(\mathrm{MH}^{+}\right)$; Anal. calcd for $\mathrm{C}_{34} \mathrm{H}_{35} \mathrm{~N}_{5} \mathrm{O}_{4}$ : C 70.69, H 6.11, N 12.12; found C 70.52, H $6.02, \mathrm{~N} 12.30$

17. Methyl 12-cyano-12-demethylpyropheobide- $a$ 8: 2,4,6-Trichloro-[1,3,5]triazine ( $370 \mathrm{mg}, 10.0 \mathrm{mmol})$ was added to DMF (2 $\mathrm{mL}$ ), maintained at $25^{\circ} \mathrm{C}$. After the formation of a white solid, the reaction was monitored (TLC) until complete disappearance of TCT, Then aldehydeoxime $7(115 \mathrm{mg}, 0.199 \mathrm{mmol})$ in DMF (5 $\mathrm{mL}$ ) was added. After the addition, the mixture was stirred at room temperature, monitored (TLC) until completion $(30 \mathrm{~h})$. Water $(20 \mathrm{~mL})$ was added then the organic phase washed with 15 $\mathrm{mL}$ of a saturated solution of $\mathrm{Na}_{2} \mathrm{CO}_{3}$, followed by $1 \mathrm{~N} \mathrm{HCl}$ and brine. The organic layer was dried $\left(\mathrm{Na}_{2} \mathrm{SO}_{4}\right)$ and vaporated in vacuo to dryness. The residue was purified on chromatographe on a silica gel column with hexane-ethyl acetate (3:1) to give 8 (69 $\mathrm{mg}, 62 \%)$ as a green solid. UV-vis $\left(\mathrm{CHCl}_{3}\right) \lambda_{\max }: 401$ (relative intensity, 1.00), $518(0.05), 564(0.22), 614(0.06), 670(0.28) \mathrm{nm}$; ${ }^{1} \mathrm{H}$ NMR $\left(\mathrm{CDCl}_{3}\right) \delta-0.49,0.72($ each br s, $2 \mathrm{H}, \mathrm{NH}), 1.42(\mathrm{t}, J=$ $\left.7.6 \mathrm{~Hz}, 3 \mathrm{H}, 8 \mathrm{~b}-\mathrm{CH}_{3}\right), 1.80\left(\mathrm{~d}, J=7.3 \mathrm{~Hz}, 3 \mathrm{H}, 18-\mathrm{CH}_{3}\right), 2.13-2.23$, 2.33-2.43, 2.54-2.66 (each $\mathrm{m}$, all $4 \mathrm{H}, 17 \mathrm{a}-\mathrm{H}+17 \mathrm{~b}-\mathrm{H}$ ), 2.80, 3.24, 3.67 (each s, each $\left.3 \mathrm{H}, \mathrm{CH}_{3}+\mathrm{OCH}_{3}\right), 3.16(\mathrm{q}, J=7.6 \mathrm{~Hz}, 2 \mathrm{H}, 8 \mathrm{a}-$ H), $4.03(\mathrm{td}, J=9.4,1.9 \mathrm{~Hz}, 1 \mathrm{H}, 17-\mathrm{H}), 4.27(\mathrm{dq}, J=7.4,1.9 \mathrm{~Hz}$, $1 \mathrm{H}, 18-\mathrm{H}), 4.85\left(\mathrm{~d}, J=19.9 \mathrm{~Hz}, 1 \mathrm{H}, 13^{2}-\mathrm{H}\right), 5.01(\mathrm{~d}, J=19.6 \mathrm{~Hz}$, $\left.1 \mathrm{H}, 13^{2}-\mathrm{H}\right), 6.15(\mathrm{~d}, J=11.6 \mathrm{~Hz}, 1 \mathrm{H}$, cis-3b-H), $6.19(\mathrm{~d}, J=17.9$ $\mathrm{Hz}, 1 \mathrm{H}$, trans-3b-H), 7.70 (dd, $J=17.9,11.6 \mathrm{~Hz}, 1 \mathrm{H}, 3 \mathrm{a}-\mathrm{H}), 8.18$, 8.51, 8.63 (each s, each $1 \mathrm{H}$, meso-H); EI-MS $m / z: 560.3\left(\mathrm{MH}^{+}\right.$); Anal. calcd for $\mathrm{C}_{34} \mathrm{H}_{33} \mathrm{~N}_{5} \mathrm{O}_{3}$ : C 72.97, H 5.94, N 12.51; found C 72.82, H 6.02, N 12.37 .

18. Tamiaki, H.; Miyatake, S.; Kureishi, Y.; Yanikaga, R. Tetrahedron 1996, 52, 12421-12432. 